\title{
The relationship between the field-shifting phenomenon and representational coherence of place cells in CA1 and CA3 in a cue-altered environment
}

\author{
Inah Lee ${ }^{1,3}$ and James J. Knierim² \\ ${ }^{1}$ Department of Psychology, University of lowa, lowa City, lowa 52242, USA; ${ }^{2}$ Department of Neurobiology and Anatomy, \\ W.M. Keck Center for the Neurobiology of Learning and Memory, University of Texas Medical School at Houston, \\ Houston, Texas 77225, USA
}

\begin{abstract}
Subfields of the hippocampus display differential dynamics in processing a spatial environment, especially when changes are introduced to the environment. Specifically, when familiar cues in the environment are spatially rearranged, place cells in the CA3 subfield tend to rotate with a particular set of cues (e.g., proximal cues), maintaining a coherent spatial representation. Place cells in $\mathrm{CAl}$, in contrast, display discordant behaviors (e.g., rotating with different sets of cues or remapping) in the same condition. In addition, on average, CA3 place cells shift their firing locations (measured by the center of mass, or COM) backward over time when the animal encounters the changed environment for the first time, but not after that first experience. However, CAl displays an opposite pattern, in which place cells exhibit the backward COM-shift only from the second day of experience, but not on the first day. Here, we examined the relationship between the environment-representing behavior (i.e., rotation vs. remapping) and the COM-shift of place fields in CA1 and CA3. Both in CA1 and CA3, the backward (as well as forward) COM-shift phenomena occurred regardless of the rotating versus remapping of the place cell. The differential, daily time course of the onset/offset of backward COM-shift in the cue-altered environment in CA1 and CA3 (on day 1 in CA1 and from day 2 onward in CA3) stems from different population dynamics between the subfields. The results suggest that heterogeneous, complex plasticity mechanisms underlie the environment-representating behavior (i.e., rotate/remap) and the COM-shifting behavior of the place cell.
\end{abstract}

It is widely recognized that the hippocampus plays a crucial role in remembering spatial contexts and past events $\left(\mathrm{O}^{\prime}\right.$ Keefe and Nadel 1978; O'Keefe and Speakman 1987; Moser et al. 1995; Vargha-Khadem et al. 1997; Lee and Kesner 2002). The existence of "place cells" (O'Keefe and Dostrovsky 1971) in the hippocampus has been considered significant physiological evidence for such a role. Place cells increase their firing rates in association with specific locations in a spatial environment, and they are considered the building blocks of a "cognitive map" of the environment, necessary not only for some forms of navigation but also for providing a spatial framework used to organize the items and events of experience (O'Keefe and Nadel 1978). The neural systems for representing a spatial environment must be flexible since an environment often does not remain constant. For example, individual items in a certain environment may be laid out differently to give a different look to the original environment. In contrast, two different environments that are located in different places may have similar appearances, due to similar individual items between the environments and/or similar geometric characteristics. How the hippocampus and its associated areas flexibly cope with such environmental variability is, therefore, a theoretically important issue.

One of the dynamic properties of the place cell is that its

\footnotetext{
${ }^{3}$ Corresponding author.
}

E-mail inah-lee@uiowa.edu; fax (319) 335-0191.

Article is online at http://www.learnmem.org/cgi/doi/10.1101//m.706207. preferred firing location (measured by its center of mass, or COM) shifts in the direction opposite to the rat's movement as the animal repeatedly traverses the same location unidirectionally. This phenomenon, first reported by Mehta et al. $(1997,2000)$ for CA1 place cells, has been interpreted as an experience-dependent plasticity mechanism in the hippocampus for learning specific spatiotemporal sequences of discrete locations, as originally proposed by computational models (Levy 1989; Blum and Abbott 1996). These models suggested that the temporally asymmetric nature of LTP induction (i.e., the presynaptic cell must fire before the postsynaptic cell) causes the formation of forward associations between place cells in a sequence, resulting in the COMshift phenomenon.

The COM-shift phenomenon recurs whenever the rat is exposed to the same, familiar environment (Mehta et al. 1997). That is, after a sufficient amount of time has passed $(<24 \mathrm{~h})$ since the last session in which the COM-shift occurred, the CA1 place fields "reset" back to their original locations at the beginning of a session and display the COM-shift pattern anew (Mehta et al. 1997, 2000; Knierim 2002; Yu et al. 2006). Explaining this reset phenomenon on the basis of the formation of forward associations between neurons, as in previous models (Blum and Abbott 1996; Mehta et al. 2000), requires the assumption that the associations are short-lived or are actively reversed by LTD overnight. The reset phenomenon thus questions the validity of interpreting the backward COM-shift as a long-term learning mechanism for the spatiotemporal sequences that occur in a given environ- 
ment, because it is difficult to explain why such sequences in the same, unchanged environment need to be learned repeatedly. Alternatively, the resetting of COM in CA1 might be explained by the lap-by-lap buildup of a hypothesized "temporal context" signal in CA1 that gates the expression of the forward sequence (Hasselmo and Eichenbaum 2005). In addition to the theoretical importance from the computational perspective, it is important to know whether these plastic changes occur in subfields in the hippocampus other than CA1, for a mechanistic understanding of the information processing in the hippocampus. Do place cells in CA3, for example, exhibit the same plastic changes as CA1 place cells? What are the differences, if any, between hippocampal subfields in processing spatial information in the environment?

Inspired by computational models and behavioral studies that have suggested differential functions of hippocampal subfields (Marr 1971; Treves and Rolls 1994; McClelland and Goddard 1996; Rolls and Treves 1998; Kesner et al. 2000; Lisman and Otmakhova 2001; Lee and Kesner 2002, 2003, 2004; Kesner et al. 2004; Treves 2004; Hasselmo and Eichenbaum 2005; Lee et al. 2005; Knierim et al. 2006), Lee et al. (2004a,b) investigated the issues mentioned above with an experimental paradigm in which the relationships between environmental cues were systematically altered (Shapiro et al. 1997; Tanila et al. 1997). That is, rats were exposed alternately to familiar and cue-altered environments, and place cell responses to the environmental changes were simultaneously recorded from CA1 and CA3 as the rat traveled along an elevated circular track consuming randomly scattered food rewards. The spatial alteration in the environment was produced by rotating a set of distal cues in the behavioral testing room clockwise (CW) and a set of proximal cues on a ring track counterclockwise (CCW), thus changing the spatial relationships between proximal and distal cue-sets (Fig. 1). The place cells in

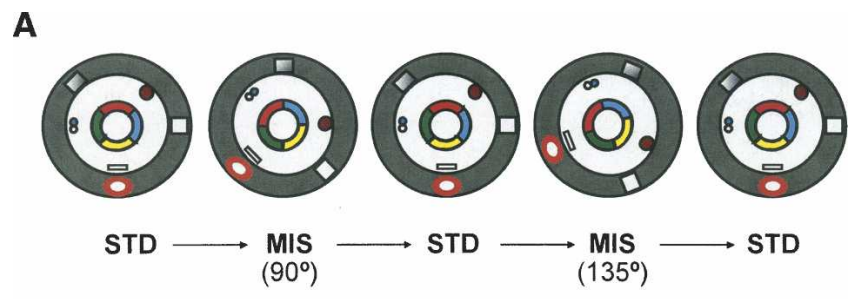

B

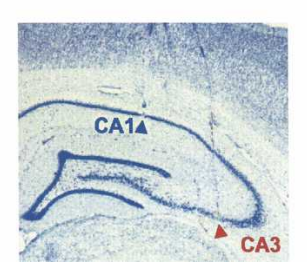

Figure 1. Experimental paradigm. $(A)$ Five recording sessions were performed in a given day in the sequence of three familiar/standard environments (STDs) interleaved with cue-altered/mismatch environments (MISs). The recording room was a curtained environment (black circular area in the figure) with distal cues placed along the curtain as well as on the floor. An elevated ring track was placed In the middle of the recording room. The track was divided into four areas, each of which was associated with a distinctive local, textured cue. The spatial relationships between distal and proximal cues were identical in STD, whereas the configural relationships between those cues were altered in MIS (to different amounts in different recording sessions). (B) Single units were recorded simultaneously from $C A 1$ and $C A 3$. CA1 recordings were from the region closest to $C A 3$, whereas $C A 3$ recordings were from the regions closest to CA1 (i.e., CA3a and CA3b). (Modified from Lee et al. 2004b).
CA1 and CA3 displayed different firing properties as the animal experienced the cue-altered environment. First, the population representation of the environment was more coherent between the original and cue-altered environment in CA3 than in CA1 (Lee et al. 2004b). The dissociation between CA1 and CA3 was less obvious when the changes made to the original environment were minimal but was noticeable as the difference became larger. Second, with respect to the backward COM-shift phenomenon, the average COM of the CA3 place fields shifted backward (i.e., opposite to the direction of the rat's motion on the track) in the first experience (i.e., day 1) of the cue-altered environment, whereas the average COM of the CA1 place fields did not display the backward shift until the next day (Lee et al. 2004a; Yu et al. 2006). This difference in the time course of the place-field plasticity suggests that the CA3 network rapidly processes the changes introduced to the environment and the CA1 network plays its role afterward.

The above-mentioned phenomena (i.e., more coherent population representation and earlier backward COM-shifting in CA3 than in CA1) were reported in separate publications (Lee et al. 2004a,b), each examined from a different analytical perspective. For example, the coherence of the population representation was investigated based on the place fields constructed by averaging the neuronal firing over multiple laps along the ring track (Lee et al. 2004b), whereas the COM-shift phenomenon was examined by comparing the location of place fields on a lap-bylap basis (Lee et al. 2004a). Here, we examine further the relationship between the differential population coherence and COM-shift phenomena of CA3 and CA1. The focus of analysis will be whether the differences in COM-shifts between CA1 and CA3 place cells were influenced by the different amounts of rotational or remapping behavior of their place fields when the environment was altered. In other words, it is possible that there is a direct relationship between place-field remapping and the COM-shift; for example, place fields that remap may not show the COM-shift effect, whereas place fields that maintain their firing fields and rotate with the cues may show the effect. If so, this relationship would tie together the two phenomena as deriving from the same underlying mechanism, as the differences between CA3 and CA1 in COM-shift may be explained by the differences between these areas in remapping. Our analyses indicate, however, that there is no such strong relationship between the two phenomena on a cell-by-cell level, showing instead that they are relatively independent phenomena that each shows a striking functional dissociation between CA3 and CA1.

\section{Results}

Previously reported dissociations between CA1 and CA3 We previously reported dissociations between CA1 and CA3 with respect to the COM-shift pattern and representational coherence as the rats experienced the cue-altered environment over multiple days (Lee et al. 2004a,b). Briefly, the experimental paradigm was as follows. Rats were exposed alternately to a familiar environment (where distal and proximal cues maintained fixed configurations) and a cue-altered environment for several days. In the cue-altered environment, the spatial relationships between distal and proximal cues were changed by rotating both proximal and distal cue-sets in opposite directions in different amounts for different recording sessions (Fig. 1A). The amount of change produced in the environment in a given recording session was operationally defined by the total angular deviation or mismatch (randomly chosen from $45^{\circ}, 90^{\circ}, 135^{\circ}$, or $180^{\circ}$ ) between the proximal and distal cues relative to their configuration in the familiar or standard environment. A brief summary of the results previously reported is provided below. 

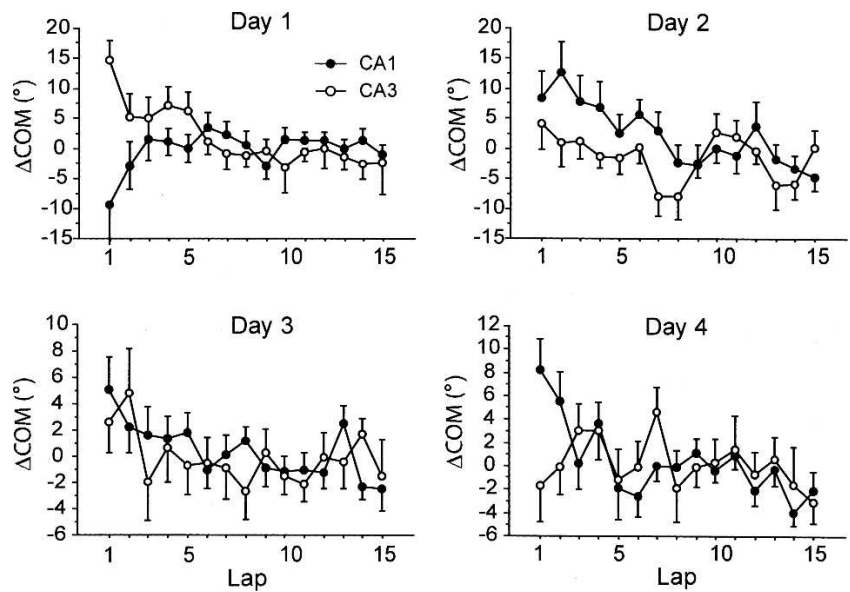

Figure 2. Backward COM-shift in CA1 and CA3 in the cue-altered environment. Across days, CA3 showed a backward COM-shift on day 1 without showing such a prominent COM-shift from day 2 onward, whereas CA1 demonstrated backward COM-shifts from day 2 onward but not on day 1. $\triangle$ COM was defined by the linear distance between the lap-based COM and the average place field-based COM. (Modified from Lee et al. 2004a).

\section{COM-shifts in CA1 and CA3 in a cue-altered environment}

Across individual days (days 1-4), CA1 and CA3 place cells recorded simultaneously (Fig. 1B) showed different patterns of COM-shifting in the cue-altered environment. Specifically, CA3 place cells moved their preferred firing positions backward on day 1 when the rats first encountered the cue-altered environment, whereas CA1 place fields did not show such a trend on day 1 (Fig. 2). However, from day 2 onward, CA1 place fields showed the backward shift each day, whereas the backward COM-shift was not obvious in CA3. Statistical tests confirmed the presence of significant COM-shifts in CA3 only on day 1 , and in CA1 only on days 2-4 (Lee et al. 2004a). The different time course for the plastic changes in CA1 and CA3 place fields suggests a functional difference between the two subfields in processing the cuealtered spatial environment; that is, CA3 rapidly processes the changes in the environment and the information processing in CA1 ensues afterward.

\section{Changes in CA1 and CA3 place fields between familiar and cue-altered environments}

The above COM-shift results were based on calculating the location of the lap-bylap COM of the place field relative to the lap-averaged COM of the overall place field within a cue-altered recording session. When the location of the place field in the cue-altered environment was compared with its location in the familiar environment, on the other hand, a different type of dissociation was obtained between CA1 and CA3 (Lee et al. 2004b). CA1 place cells displayed more heterogeneous behavior than did CA3 place cells (Fig. 3). For example, the majority of CA3 place cells rotated their fields CCW in the direction of the proximal cues on the ring track, whereas similar proportions of CA1 place cells rotated $\mathrm{CW}$ or CCW in the cue-altered environment, displayed ambiguous behaviors that did not allow easy characterization (such as splitting into multiple fields), or completely changed their activity by losing their place fields or generating new place fields. The dissociation was most prominent when the distal and proximal cues were misaligned by an amount exceeding $45^{\circ}$ (i.e., $90^{\circ}, 135^{\circ}$, and $180^{\circ}$ ). It was also robustly observed when ensembles of CA1 and CA3 neurons simultaneously recorded within subjects were compared. The results suggest that the CA3 network maintains a more coherent representation of the environment in the face of ambiguities or alterations introduced to the environment.

\section{Relationship between place cell behavior and COM-shifts}

\section{The relationship at the population level}

Considering the different proportions of place cells exhibiting remapping versus rotation between CA1 and CA3 (Fig. 3), we examined the possibility that these differences influenced the differential COM-shifts in CA1 and CA3 (using the same data sets as in Lee et al. 2004a,b). For this analysis, as previously reported (Lee et al. 2004b), various place cell behaviors in the cue-altered environment (compared to the familiar environment) were categorized into either rotation or remapping, based on visual examination. The resulting proportions of place cells showing rotation and remapping in the cue-altered environment are shown separately for CA1 and CA3 in Figure 4. Similar proportions of place cells rotated and remapped between CA1 and CA3 on the first day and also on the fourth day of exposure to the cue-altered environment [day 1: $\chi^{2}(1, N=51)=0.724, P=0.39$; day $4: \chi^{2}(1$, $N=60)=0.512, P=0.47]$, whereas more remapping occurred in CA1 than in CA3 on days 2 and 3 [day 2: $\chi^{2}(1, N=41)=10.5$, $P<0.01$; day $\left.3: \chi^{2}(1, N=76)=5.8, P<0.05\right]$. Comparison of the rotation/remapping patterns in CA1 and CA3 on day 1 with the COM-shift patterns on the same day (Fig. 2), in which a strong dissociation between CA1 and CA3 was demonstrated, suggests that the amount of remapping or rotation of the place field is unlikely to be related to the COM-shift dissociation. Furthermore, the overall rotation/remapping trends across days in each subfield do not correlate with the daily COM-shift trends. For example, in CA3, the proportions of rotating place fields were not significantly different between days 1 and $4\left[\chi^{2}(1\right.$, $N=50)=0.512, P=0.47]$, whereas there was noticeable difference in the amount of backward COM-shift between those days,

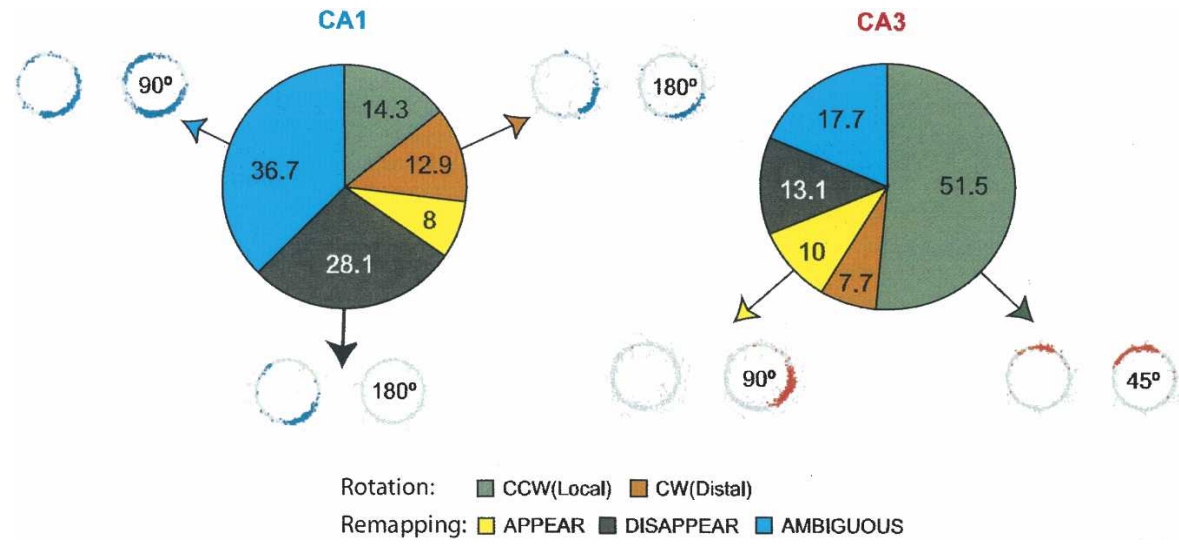

Figure 3. Classification of place field behavior in the cue-altered environment. Place cells in CA1 and CA3 rotated their fields with proximal/distal cues, generated ambiguous responses (such as splitting fields), or turned on/off fields in the cue-altered environment. The pie chart shows the proportion of cells that showed the behavior in each category and some place field examples are shown. CW indicates clockwise rotation (presumably following distal cues); CCW, counterclockwise rotation (presumably following proximal cues). (Modified from Lee et al. 2004b). 


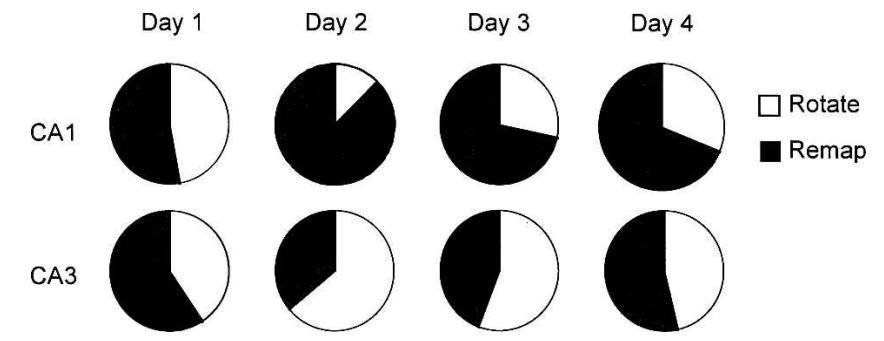

Figure 4. The proportion of cells that rotate vs. remap in CA1 and CA3 between familiar (STD) and cue-altered (MIS) environments. Only the first cue-altered recording session per day was used to assess the response to novelty. When visually inspected, place fields rotated either CW or CCW ("rotate") or their relationships to cues were less obvious (e.g., appearing, disappearing, splitting into multiple fields, etc.; "remap"). Note that the significant difference in the proportions for rotation and remapping between CA 1 and CA 3 are not systematically related to the dissociated COM-shift patterns between those subfields across days in Figure 2, suggesting a minor relationship, if any, between remapping and the COM-shift phenomena. CA1: day $1, n=14 / 29$ (rotate) and 15/29 (remap); day $2, n=2 / 16$ (rotate) and 14/16 (remap); day $3, n=12 / 42$ (rotate) and 30/42 (remap); day $4, n=10 / 32$ (rotate) and 22/32 (remap). CA3: day $1, n=8 / 22$ (rotate) and $14 / 22$ (remap); day $2, n=16 / 25$ (rotate) and 9/25 (remap); day $3, n=19 / 34$ (rotate) and 15/34 (remap); day $4, n=13 / 28$ (rotate) and 15/28 (remap). It is not clear whether the more pronounced differences between CA3 and CA1 on days 2 and 3 (compared to days 1 and 4) reflect statistical variability, a complex interaction between mismatch amount and cumulative experience with the mismatch manipulation, or some other set of unknown variables. with only day 1 showing a significant backward shift. Similarly, the proportions of rotating place fields were not different between days 1 and 4 for CA1 $\left[\chi^{2}(1, N=61)=1.85, P=0.17\right]$, yet only day 4 showed a significant COM-shift (Fig. 2).

The apparent independence of the two phenomena is further supported when the daily COM-shift graphs (Fig. 5) for each subfield in cue-altered environments were separately plotted based on the categorized place-cell behavior (i.e., rotation versus remapping). On day 1, neither cells classified as rotation nor cells classified as remapping showed a strong COM-shift in CA1. As a result, the slopes of the regression lines were neither significantly negative nor positive (linear regression; $P=0.52$ for rotation cells and $P=0.37$ for remapping cells). However, both classes of cells showed clear COM-shifts in CA3 on day 1 (linear regression; $P<0.0001$ for rotation cells and $P<0.01$ for remapping cells). For days $2-4$, the data for CA1 are variable; the slopes of regression line for the rotation cells on day 2 and day 3 were not significantly negative (linear regression, $P$-values $>0.01$ ), but the slope on day 4 was significantly negative $(P<0.0001)$. For remapping cells in CA1, regression lines for days $2-4$ were negatively sloped; significantly on days 2 and 3 ( $P$-values $<0.0001)$ and a trend on day $4(P=0.06)$. Importantly, there is no clear dichotomy between rotating and remapping cells, as the backward shift can be seen in both classes of cells. In CA3, the data are clearer, as neither rotating cells nor remapping cells showed strong evidence of a consistent COM-shift on days $2-4$ ( $P$-values $>0.1)$, whereas both types of cells exhibited significant backward shifts on day $1(P$ -
CA1
D1
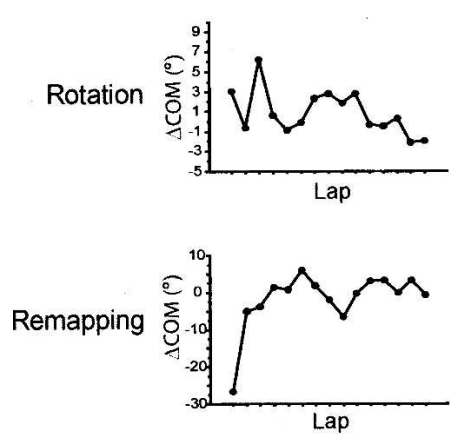

Lap
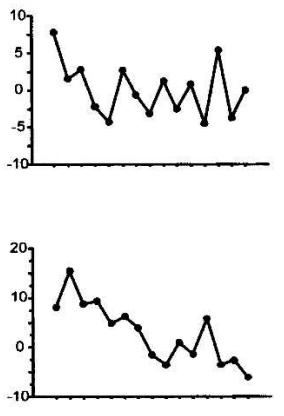

CA3

D1
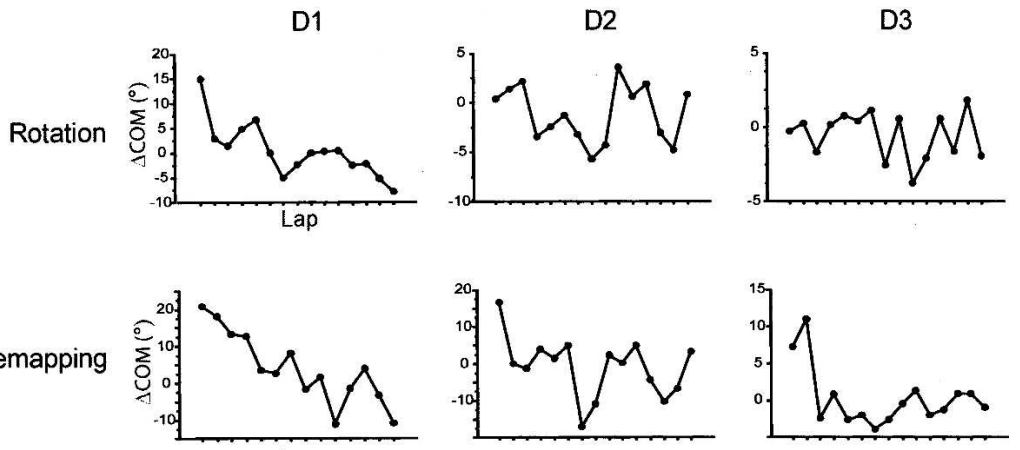

Remapping

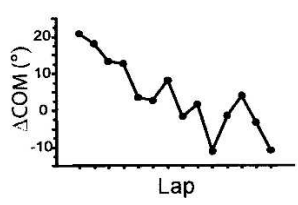

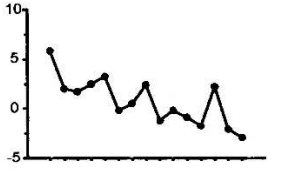

D3
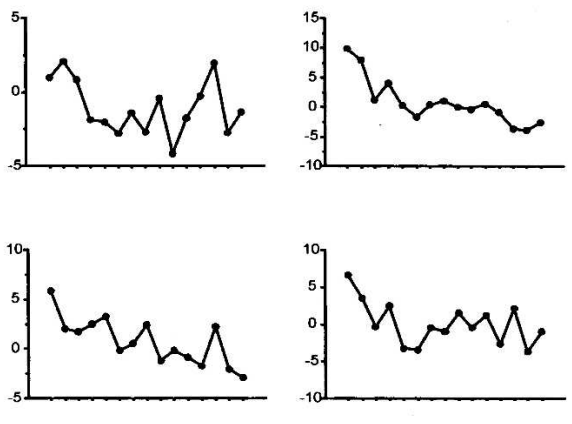

Figure 5. Relationship between place field behavior and COM shift. The average COM-shift plots were constructed for cells that were classified as remapping vs. rotating. Although the smaller numbers of cells in each group make the results more variable than the combined data, there were no obvious differences between the two groups in each subregion. $X$-axis, laps; $Y$-axis, $\Delta C O M$. 
values $<0.01)$. Although the trends across days, regions, and cell type are variable, there is no evidence that the difference between CA1 and CA3 results from different proportions of cells that rotate or remap in the two subfields.

\section{The relationship at the individual neuronal level}

The COM-shift phenomenon has so far been described at the population level, in which the COM-shifts of many place fields in a population are averaged. The original report of the COMshift phenomenon (Mehta et al. 1997) was also based on the statistical analysis of population data. It is important, however, to describe the patterns of COM-shifts in individual place cells to understand the properties of the network more thoroughly. We examined whether the differences in COM-shifts between CA1 and CA3 were reflected in all individual place fields in the regions and also whether there was a relationship between COM-shift and place-field rotation/remapping at the individual place field level. Figure 6 shows representative examples of place fields recorded between familiar and cue-altered environments and the COM-shift pattern across laps within the cue-altered environment. As shown, some place cells showed clear COM-shift patterns, but the direction of COM-shift was not always backward. That is, some place cells shifted their COMs forward across laps. When the individual COM-shift patterns were examined with the rotation/remapping behavior of place fields, there was no apparent relationship between the two phenomena. For example, different place fields that rotated CW (or CCW) displayed opposite directions of COM-shifting (Fig. 6). Based on subjective
CA1

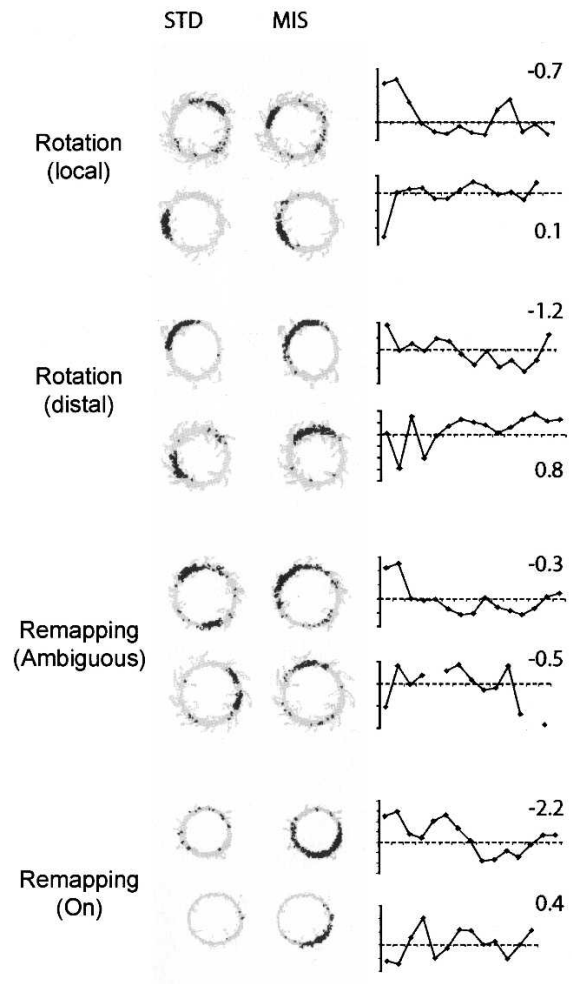

STD

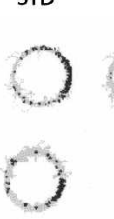

MIS
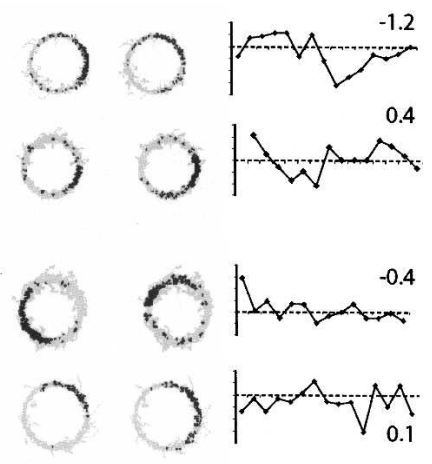

0.3
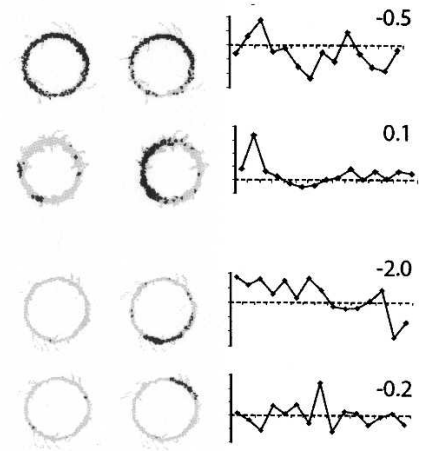

Figure 6. Relationship between place field behavior and COM-shift. COM-shift was seen in all types of cells in both CA1 and CA3, including cells that remapped their place fields and cells that did not remap. In addition, within each category of place cell behavior, some cells (upper panel of each pair) showed the backward shifting-trend in their locations, whereas the COM of other cells (lower panel of each pair) either shifted forward or remained stationary. The number associated with each graph denotes the slope of the linear regression line. $X$-axis, laps; $Y$-axis, $\triangle C O M$. visual inspections, the rotational or remapping pattern of the place field does not reliably predict the pattern of plastic changes the location of the place field across time.

rons, the slope of the COM-shift curve for an individual place cell was calculated based on the robust-regression method (using Matlab 7.4, The MathWorks, Inc.), with which the slope of the regression line was less contaminated by extreme outliers than conventional least-squares-based regression method. xamination of COM-shift patterns at the individual neuronal 1 , but these cells were matched by similar numbers of cells that ped a strong forward shift or no shift (Fig. 7). The effect, at cancelled out the forward-shift cells, and the resulting population COM-shift trend on average was flat in CA1 (Fig. 2). From day 2 onward in CA1, however, the backward COM-shift pattern we population of place cells (Fig. 7), and the shifting (Fig. 2). This trend is confirmed when the distribution of the slopes of individual place field COM-shifts was obtained for each day's cue-altered condition (Fig. 8); the proportions of place day 1 (one-sample sign test, $P>0.5$ ) but were significantly different from day 2 onward (one-sample sign test, $P$-values $<0.001$ for day 2 and day $4, P<0.05$ for day 3 ). In CA3, contrary to CA1, the dominant pattern of COM-shift on day 1 was backward shifting (Fig. 7). From day 2 onward, it appears that the COM of most place fields remained stationary across laps (only fluctuating around the horizontal axis of the COM-shift graphs, thus neither shifting forward nor backward) compared with day 1 . The histograms of the slopes of the COM-shift curves of CA3 place cells indicate that there were more negatively sloped COMshifting cells only on day 1 (one-sample sign test, $P=0.01$ ), but not in days $2-4$ $(P$-values $>0.1)$.

It is important to note, therefore, that there appears to be two different mechanisms at work in CA3 and CA1 when their respective population averages show no backward COM-shift. In CA1 on day 1 , individual cells show strong forward and backward shifts that cancel each other out, which is manifested as the similar numbers of place fields with negative and positive slope in the histogram (Fig. 8). However, in CA3 on days 2-4 when the population COMshifts were not observed, the COM-shift slopes of most fields were distributed more narrowly around zero (i.e., stationary COM across laps) in the histograms (Fig. 8). This distribution suggests that individual CA3 fields remained stable and coherent, with little forward or backward shifts, in days 2-4. As described in Lee et al. (2004a), the CA3 place fields on the latter days are nega- 


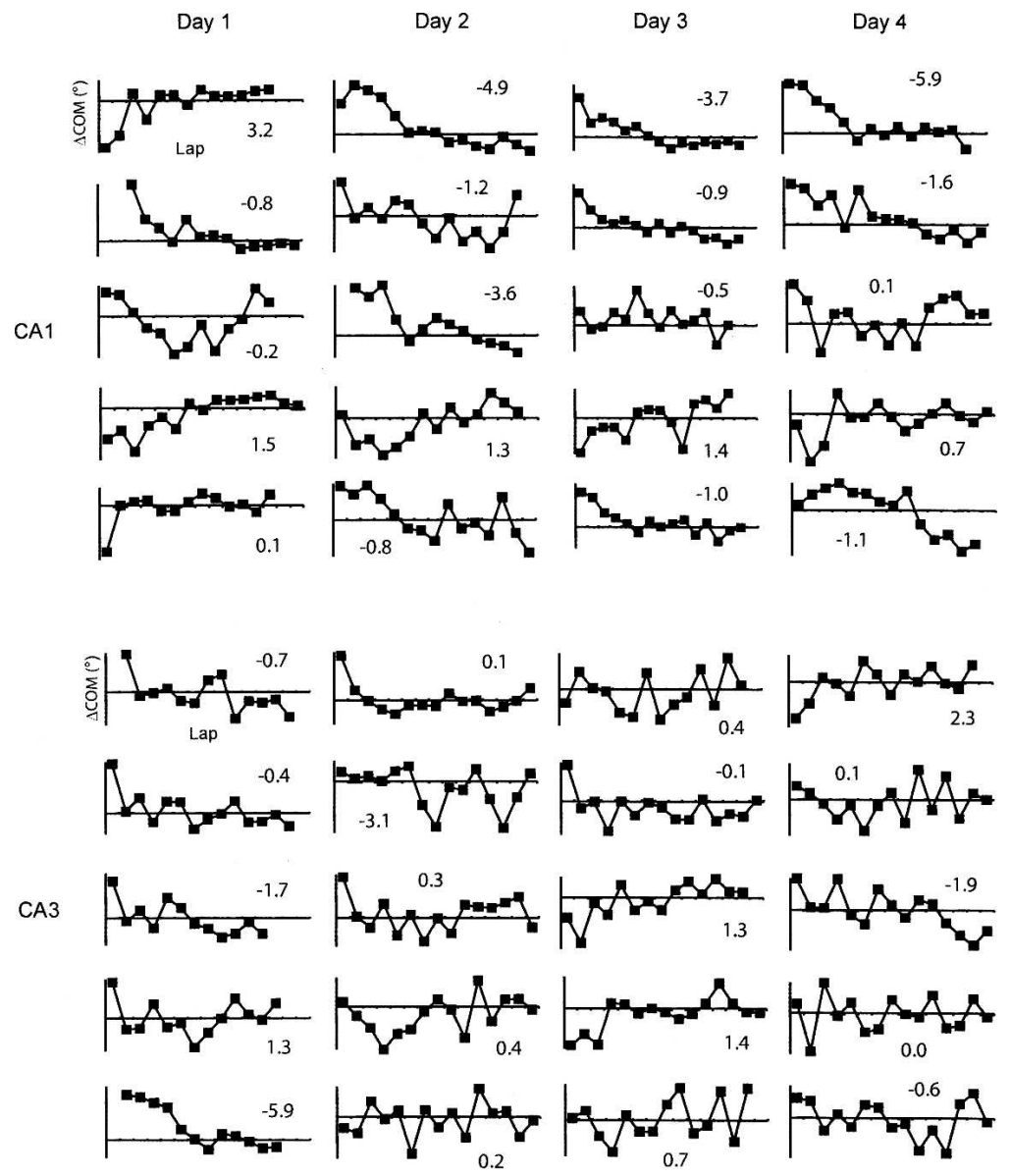

Figure 7. Examples of the daily COM-shift patterns across laps in CA1 and CA3 place cells recorded in the cue-altered environment (cells were not simultaneously recorded). The number associated with each graph denotes the slope of the linear regression line. $X$-axis, laps; $Y$-axis, $\Delta C O M$.

tively skewed from the first lap onward in these sessions, supporting the interpretation that the lack of a COM-shift in CA3 is the consequence of the place fields maintaining their shifted COMs across days after the initial COM-shift on day 1.

\section{Discussion}

The backward COM-shift of place fields was first reported in CA1 when the rat ran stereotyped routes on a track. In the original reports (Mehta et al. 1997, 2000), it was also noted that the CA1 place field expanded backward and became negatively skewed as the animal experienced the same environment repeatedly. The backward shift was abolished by NMDA-receptor blockade and was diminished in aged rats (Shen et al. 1997; Ekstrom et al. 2001). Collectively, these phenomena led researchers to hypothesize that place fields representing adjacent locations are associated with each other spatiotemporally, thus enabling the animal to learn the sequential spatial information as predicted by computational models (Levy 1989; Blum and Abbott 1996; Mehta et al. 2000; Hasselmo and Eichenbaum 2005). In our study, only CA3 place cells recorded on day 1 in the cue-altered environment exhibited the changes reported by the prior studies (Mehta et al. 2000). In later days, however, CA3 place cells were skewed negatively from the onset of the recording session in the cue-altered environment (Lee et al. 2004a) and did not display the backward shift of the COM. In contrast, although CA1 place cells showed backward COM-shifts in the cue-altered environment from day 2 onward, the development of negative skewness in the place field account for some of the complexity in the experimental results.

It is well known that the hippocampus plays a critical role in processing novel stimuli or newly arranged, familiar stimuli (Myhrer 1988; Knight 1996; Stern et al. 1996; Shapiro et al. 1997; Tanila et al. 1997; Grunwald et al. 1998; Honey et al. 1998; Wood et al. 1999; Lisman and Otmakhova 2001; Vinogradova, 2001; Lee and Kesner 2002; Mumby et al. 2002; Lee et al. 2004b, 2005; Hunsaker et al. 2007). Although the functional significance of the COM-shift phenomenon is currently unclear, the differential amount and pattern of plastic changes in the locations of place fields (as well as their shapes) over time between CA1 and CA3 certainly suggest that the hippocampal networks are engaged in dynamic information processing as the animal experiences a novel/changed environment. Specifically, our study shows that place cells both in CA1 and CA3 manifest dynamic shifting of their locations across laps as the animal encounters the cuealtered environment for the first time on day 1 . However, as shown in Figures 7 and 8, CA3 place cells exhibit more coherent shifting behavior (backward COM-shift as well as the development of negative skewness) on day 1 than the place cells in CA1 (which display similar proportions of backward and forward COM-shifting cells). Interestingly, after the first encounter with the cue-altered environment on day 1, CA3 cells did not show such dynamic plasticity on subsequent days (as if the subfield was no longer "interested" in the cue-altered environment), whereas CA1 place cells continued to show plastic changes in their firing properties from day 2 onward (importantly, the pat- 
CA 1

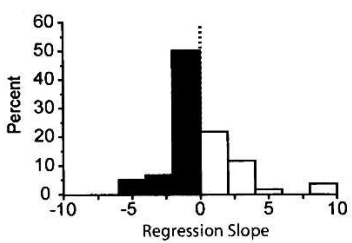

Day 1

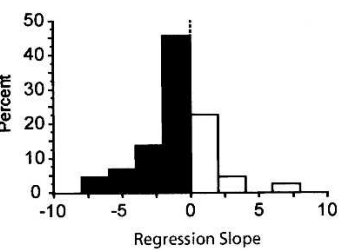

Day 2

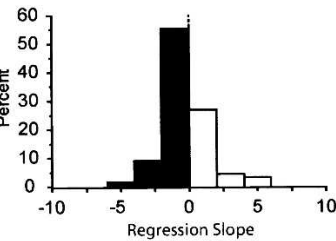

Day 3

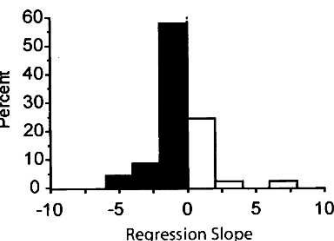

CA3
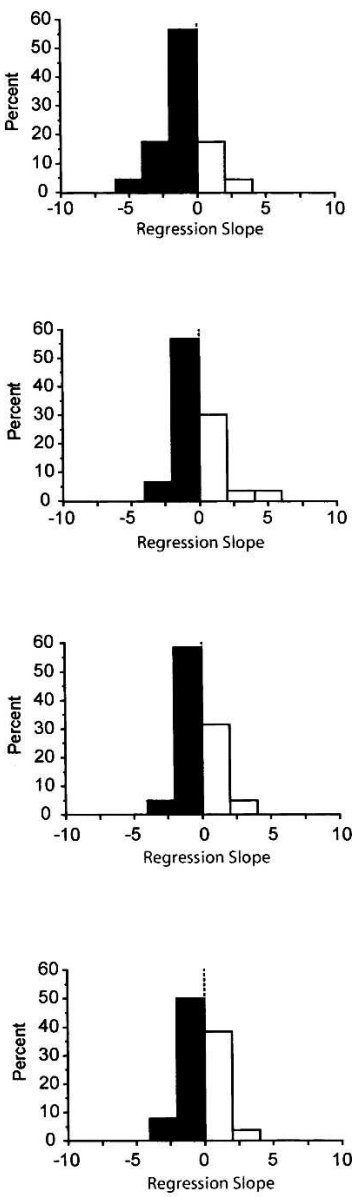

Figure 8. Daily distribution of COM-shift directions in CA1 and CA3 place cells in the cue-altered environment. Each histogram represents the number of place fields that showed backward COM-shift (black) versus forward COM-shift (white).

tern of shifts across cells was more coherent than on day 1 , resulting in an overall backward COM-shift). It is not clear why CA3 place fields do not shift backward in familiar environments and in the second mismatch session onward. One possibility is that the cellular plasticity mechanisms in CA3 are activated strongly only during the first exposure to a novel or altered environment, suggesting a specialized role for CA3 plasticity only in novel situations. An alternative is that the synaptic strengthening in CA3 simply becomes saturated during the initial experience, and the place fields shift and skew backward to a maximum extent that is then maintained across many days.

The CA3 subfield's capability of producing a coherent pattern of changes in response to a newly encountered, changed environment was also identified in our previous study (Lee et al. 2004b). This capability of CA3 is presumably due to the existence of recurrent collaterals in CA3 (but not in CA1) and may explain prior lesion/pharmacological studies that showed disrupted performance of the rats with selective damage in CA3 when familiar environments were changed (Lee and Kesner 2002; Nakazawa et al. 2002; Gold and Kesner 2005; Lee et al. 2005), when learning occurred in a novel environment (Lee and Kesner 2003, 2004), or when goal locations in a familiar environment were changed every day (Nakazawa et al. 2003). That is, without CA3, the hippocampal efferents may carry only discordant information when the animal first experiences a novel environment, similar to the inharmonious population responses observed in CA 1 on day 1 in the current study (Fig. 8; see also Lee et al. 2004b). This may lead to the impairment of the CA3-lesioned animals in producing a coherent cognitive framework within which rapid learning can take place (Tse et al. 2007).

Interestingly, CA1 is capable of producing a coherent backward shift, but only after a delay (from day 2). It is possible that the coherence of CA1 requires prior formation of cell assemblies in upstream regions (e.g., CA3), with perhaps a subsequent period of slow-wave sleep or quiet restfulness to allow CA3 to train the CA1 network and impose the new assembly structure on the CA1 cells during the sharp wave events associated with these epochs (Wilson and McNaughton 1994; Skaggs and McNaughton 1996; Lee and Wilson 2002; Jackson et al. 2006). This delaydependent involvement of the CA1 network has also been reported in lesion/pharmacological studies; for example, in a delayed match-to-place task, Lee and colleagues have shown that animals with localized lesions in CA1 (Lee and Kesner 2003) or with inactivation of NMDA receptors in CA1 (Lee and Kesner 2002) are impaired in remembering the location visited during a sample phase only when the delay is significantly longer (e.g., 5 min) but are normal when the delay is short (e.g., $10 \mathrm{sec}$ ). In a contextual fear-conditioning task, CA1-lesioned animals were significantly impaired in retrieving contextual memory $24 \mathrm{~h}$ after learning, whereas CA3-lesioned animals were only impaired at the initial stage of the acquisition of the contextual memory but not during the retrieval in $24 \mathrm{~h}$ (Lee and Kesner 2004). These data support the notion of a dissociation between CA3 and CA1 in terms of short-term versus intermediate-term memory (Rolls and Kesner 2006).

The aim of this study was to determine whether the differences between CA1 and CA3 in the backward shift phenomena (Fig. 3) could be explained straightforwardly by the differences in these two regions in the degree of remapping in the same experiment (Fig. 2). The results suggest that knowing whether a place field rotates or remaps in the cue-altered environment provides little information on whether the place field displays a COMshift in that environment. Nonetheless, it may be possible to tie together the results of Figure 2 and Figure 3 at a more global level related to the tendency of the CA3 network to respond to environmental alterations in a more coherent manner than CA1, presumably because of the recurrent collateral system of CA3. This tendency would explain both the increased coherence of CA3 representations compared with CA1 representations (i.e., in CA3, a majority of place fields rotate with the local cues whereas only a minority remap, and in CA1 a majority remap and only a minority rotate in a split representation; Fig. 3) and the differential COM-shift on day 1 of the mismatch session (i.e., CA3 place fields more coherently shift backward, whereas CA1 place fields split between forward and backward-shifting place fields; Figs. 2, 8). The COM-shift differences between CA3 and CA1 from day 2 onward may arise from a different set of mechanisms (e.g., the delayed coherence of the CA1 response and the lack of further plasticity in CA3 because of the long-term storage of the synaptic changes from day 1). Providing more mechanistic remarks on the relationship between remapping and COM-shifting is beyond the scope of what the current experimental paradigm can offer mainly because the paradigm itself was not designed to test such a relationship. Furthermore, the functional significance of both the COM-shift phenomenon and the remapping phenomenon are still not well understood (O'Keefe and Speakman 1987; Barnes et al. 1997; Oler and Markus 2000; Jeffery et al. 2003; Knierim 2003). Future studies focusing more on the functional significance of these two phenomena may shed light on the relationship between the two and the underlying plasticity mechanisms. 


\section{Materials and Methods}

\section{Subjects}

Five male Long-Evans rats were maintained at $80 \%-90 \%$ of their ad libitum weights and had free access to water. The rats were housed individually on a reversed 12:12 light/dark cycle, and all the experiments were performed during the dark portion of the cycle. Animal care and surgical procedures were performed according to National Institutes of Health guidelines and were approved by the University of Texas Health Science Center at Houston Institutional Animal Care and Use Committee.

\section{Surgery}

Each animal was anesthetized (initial dose of $60 \mathrm{mg} / \mathrm{kg}$ ketamine and $8 \mathrm{mg} / \mathrm{kg}$ xylazine, followed by isoflurane inhalation to effect during surgery), and a microdrive array was implanted over the right dorsal hippocampus ( $4.2 \mathrm{~mm}$ posterior to bregma; $4.5 \mathrm{~mm}$ lateral from midline). The microdrive array was a custom-built hyperdrive composed of 20 recording probes. Recording probes were tetrodes (Wilson and McNaughton 1993), made of four lengths of fine nichrome wire (Rediohm-800, 0.0127 mm; Kanthal) twisted together and gold-plated to reduce the final impedance to 250-500 k $\Omega$ measured at $1 \mathrm{kHz}$ (Impedance tester IMP-1; BAK Electronics). For detailed descriptions of the recording system, see Knierim (2002).

\section{Apparatus and behavioral training}

Following a week of recovery after surgery, the rats were trained to run $\mathrm{CW}$ on a circular track $(56-\mathrm{cm}$ inner diameter and $76-\mathrm{cm}$ outer diameter) to collect chocolate sprinkles arbitrarily placed on the track by an experimenter. The rats underwent six to 21 training sessions over $\sim 7 \mathrm{~d}$ (range, 2-12 d) before the beginning of the experiment. The track was positioned inside a black circular curtain (2.7-m diameter) that reached from the ceiling to the floor. Six different objects (distal cues) were hanging on the curtain or standing on the floor at the perimeter of the curtain (hanging cues were a brown cardboard circle, a black-and-white striped card, and a white card; standing cues were a white box, an intravenous stand with a laboratory coat and a blue cloth, and a roll of brown wrapping paper.) The track itself was composed of four different textured surfaces, each covering one-quarter of the ring: a gray rubber mat with a pebbled surface, brown mediumgrit sand paper, beige carpet-pad material, and gray duct tape with white tape stripes. Throughout all training sessions, the local cues on the track and the array of distal cues were maintained at a constant configuration (standard session). During the training days, the electrodes were advanced gradually over the course of many days to place the electrodes in the vicinity of pyramidal cell layers in CA1 and CA3.

On each testing day, each animal was given a baseline sleep period (30 min), during which multiple cells were recorded, before the first behavioral testing session and after the last testing session. The data that were collected during sleep were used to determine the stability of recordings that were made during behavioral sessions, and unstable cells were not analyzed further. After the first sleep session, the rat was placed in a covered box and was walked briefly around the computer room before entering the adjacent, behavioral testing room. The rat was placed on the ring track at an arbitrarily chosen location and finished 15 CCW laps for a given session. On a given day, three standard cue configuration sessions were presented. In between those identical standard sessions, the track was rotated $22.5^{\circ}, 45^{\circ}, 67.5^{\circ}$, or $90^{\circ}$, and the distal cues were rotated CW by an equal amount (mismatch session) (Fig. 1A). The total amount of mismatch between local and distal cues, therefore, was $45^{\circ}, 90^{\circ}, 135^{\circ}$, or $180^{\circ}$. The rats experienced two complete sets of each mismatch amount over $4 \mathrm{~d}$, with each mismatch being run in a pseudorandom order.

\section{Histology}

After the completion of experiments, small marker lesions were made on a subset of the tetrode tips $1 \mathrm{~d}$ before perfusion. Each animal was perfused transcardially. The frozen brain was cut at 40-um sections on either a microtome or a cryostat and stained with Cresyl violet. Electrode tracks were identified under a light microscope. Each tetrode was then assigned to a hippocampal subfield based on the histological results in addition to the electrophysiological depth profiles that were collected during the experiments. Specifically, digital photomicrographs were taken for all serial sections of the hippocampus $(40 \mu \mathrm{m})$. Tetrode tracks were traced on the digital images using graphic software (Adobe), and those retouched images were reconstructed threedimensionally (Voxar). Microscopic examinations were used in parallel in the course of reconstruction. Rotated threedimensional views of the reconstructed three-dimensional image were compared to the configuration of the tetrodes in the original tetrode bundle for accurate identification of the tetrodes.

\section{Data analysis}

Details of data analysis methods can be found in our previous studies (Lee et al. 2004a,b). Briefly, offline unit isolation was made using custom software running on a personal computer. The relative amplitudes of signals that were recorded simultaneously at four different wires of the tetrode were primarily used for the isolation of single units. Other parameters of waveforms, such as spike width and height, were also used. Recording stability was assessed visually by comparing patterns of waveform parameter clusters in the two sleep sessions before and after the behavioral sessions each day.

The circular ring track was linearized for the purpose of analysis. The track was divided into 360 bins $\left(1^{\circ} \mathrm{bin}\right)$, and a firing rate for each bin was calculated by dividing the number of spikes that were fired while the rat occupied that bin by the amount of time that was spent in the bin. Following the method of Mehta et al. (1997), the place field boundaries were defined by the bins in which the mean firing rate fell below $10 \%$ of the peak firing rate of the place field for 20 contiguous bins (a lap-based place field was subsequently analyzed within these boundaries only). The spatial information score was calculated according to the method of Skaggs et al. (1993; 1996). Only cells that had a statistically significant information score of $\geq 0.5$ with $\geq 50$ spikes were included in the analysis. The average position of a place field on the track was defined by calculating the center of mass (COM) of the firing rate distribution within the place field boundaries (Mehta et al. 1997, 2000). Calculation of the lapbased COM was restricted to the laps in which at least two spikes fired. $\triangle \mathrm{COM}$ was defined by the linear distance between the lapbased COM and the average place field-based COM.

Classification of place field behavior in response to the changes in the environment was performed by visually inspecting the place fields between the familiar environment and cuealtered conditions; place cells rotated either CW or CCW ("rotate") or produced less obvious behaviors (e.g., appearing, disappearing, splitting into multiple fields, etc.; "remap"). Two observers classified the cells independently (both being blind to the subfields the cells belong to), and both observers produced classifications with similar proportions of cells in each category.

\section{Acknowledgments}

We thank Geeta Rao and D. Yoganarasimha for assistance in data collection. This work was supported by grants R01 NS39456 and K02 MH63297 from the National Institutes of Health for J.J.K. and the Pickwick fellowship from the National Sleep Foundation for I.L.

\section{References}

Barnes, C.A., Suster, M.S., Shen, J., and McNaughton, B.L. 1997. Multistability of cognitive maps in the hippocampus of old rats. Nature 388: 272-275.

Blum, K.I. and Abbott, L.F. 1996. A model of spatial map formation in the hippocampus of the rat. Neural Comput. 8: 85-93.

Ekstrom, A.D., Meltzer, J., McNaughton, B.L., and Barnes, C.A. 2001 Nmda receptor antagonism blocks experience-dependent expansion of hippocampal "place fields." Neuron 31: 631-638. 
Gold, A.E. and Kesner, R.P. 2005. The role of the CA3 subregion of the dorsal hippocampus in spatial pattern completion in the rat. Hippocampus 15: 808-814.

Grunwald, T., Lehnertz, K., Heinze, H.J., Helmstaedter, C., and Elger, C.E. 1998. Verbal novelty detection within the human hippocampus proper. Proc. Natl. Acad. Sci. 95: 3193-3197.

Hasselmo, M.E. and Eichenbaum, H. 2005. Hippocampal mechanisms for the context-dependent retrieval of episodes. Neural Netw. 18: $1172-1190$.

Honey, R.C., Watt, A., and Good, M. 1998. Hippocampal lesions disrupt an associative mismatch process. J. Neurosci. 18: 2226-2230.

Hunsaker, M.R., Rogers, J.L., and Kesner, R.P. 2007. Behavioral characterization of a transection of dorsal CA3 subcortical efferents: Comparison with scopolamine and physostigmine infusions into dorsal CA3. Neurobiol. Learn. Mem. 88: 127-136.

Jackson, J.C., Johnson, A., and Redish, A.D. 2006. Hippocampal sharp waves and reactivation during awake states depend on repeated sequential experience. J. Neurosci. 26: 12415-12426.

Jeffery, K.J., Gilbert, A., Burton, S., and Strudwick, A. 2003. Preserved performance in a hippocampal-dependent spatial task despite complete place cell remapping. Hippocampus 13: 175-189.

Kesner, R.P., Gilbert, P.E., and Wallenstein, G.V. 2000. Testing neural network models of memory with behavioral experiments. Curr. Opin. Neurobiol. 10: 260-265.

Kesner, R.P., Lee, I., and Gilbert, P. 2004. A behavioral assessment of hippocampal function based on a subregional analysis. Rev. Neurosci. 15: $333-351$

Knierim, J.J. 2002. Dynamic interactions between local surface cues, distal landmarks, and intrinsic circuitry in hippocampal place cells J. Neurosci. 22: 6254-6264.

Knierim, J.J. 2003. Hippocampal remapping: Implications for spatial learning and navigation. In The neurobiology of spatial behaviour (ed. K.J. Jeffery), pp. 226-239. Oxford University Press, Oxford, UK.

Knierim, J.J., Lee, I., and Hargreaves, E.L. 2006. Hippocampal place cells: Parallel input streams, subregional processing, and implications for episodic memory. Hippocampus 16: 755-764.

Knight, R. 1996. Contribution of human hippocampal region to novelty detection. Nature 383: 256-259.

Lee, I. and Kesner, R.P. 2002. Differential contribution of NMDA receptors in hippocampal subregions to spatial working memory. Nat. Neurosci. 5: 162-168.

Lee, I. and Kesner, R.P. 2003. Differential roles of dorsal hippocampal subregions in spatial working memory with short versus intermediate delay. Behav. Neurosci. 117: 1044-1053.

Lee, I. and Kesner, R.P. 2004. Differential contributions of dorsal hippocampal subregions to memory acquisition and retrieval in contextual fear-conditioning. Hippocampus 14: 301-310.

Lee, A.K. and Wilson, M.A. 2002. Memory of sequential experience in the hippocampus during slow wave sleep. Neuron 36: 1183-1194.

Lee, I., Rao, G., and Knierim, J.J. 2004a. A double dissociation between hippocampal subfields: differential time course of CA3 and CA1 place cells for processing changed environments. Neuron 42: $803-815$.

Lee, I., Yoganarasimha, D., Rao, G., and Knierim, J.J. 2004b. Comparison of population coherence of place cells in hippocampal subfields CA1 and CA3. Nature 430: $456-459$.

Lee, I., Hunsaker, M.R., and Kesner, R.P. 2005. The role of hippocampal subregions in detecting spatial novelty. Behav. Neurosci. 119: $145-153$.

Lee, I., Griffin, A.L., Zilli, E.A., Eichenbaum, H., and Hasselmo, M.E. 2006. Gradual translocation of spatial correlates of neuronal firing in the hippocampus toward prospective reward locations. Neuron 51: $639-650$.

Levy, W.B. 1989. A computational approach to hippocampal function. In Computational models of learning in simple neural systems (eds. R.D Hawkins and G.H. Bower), pp. 243-305. Academic Press, Orlando, FL.

Lisman, J.E. and Otmakhova, N.A. 2001. Storage, recall, and novelty detection of sequences by the hippocampus: Elaborating on the SOCRATIC model to account for normal and aberrant effects of dopamine. Hippocampus 11: 551-568.

Marr, D. 1971. Simple memory: A theory for archicortex. Philos. Trans. R. Soc. Lond. B Biol. Sci. 262: 23-81.

McClelland, J.L. and Goddard, N.H. 1996. Considerations arising from a complementary learning systems perspective on hippocampus and neocortex. Hippocampus 6: 654-665.

Mehta, M.R., Barnes, C.A., and McNaughton, B.L. 1997. Experience-dependent, asymmetric expansion of hippocampal place fields. Proc. Natl. Acad. Sci. 94: 8918-8921.

Mehta, M.R., Quirk, M.C., and Wilson, M.A. 2000. Experience-dependent asymmetric shape of hippocampal receptive fields. Neuron 25: 707-715.
Moser, M.B., Moser, E.I., Forrest, E., Andersen, P., and Morris, R.G. 1995. Spatial learning with a minislab in the dorsal hippocampus. Proc. Natl. Acad. Sci. 92: 9697-9701.

Mumby, D.G., Gaskin, S., Glenn, M.J., Schramek, T.E., and Lehmann, H. 2002. Hippocampal damage and exploratory preferences in rats: Memory for objects, places, and contexts. Learn. Mem. 9: 49-57.

Myhrer, T. 1988. Exploratory behavior and reaction to novelty in rats with hippocampal perforant path systems disrupted. Behav. Neurosci. 102: $356-362$.

Nakazawa, K., Quirk, M.C., Chitwood, R.A., Watanabe, M., Yeckel, M.F., Sun, L.D., Kato, A., Carr, C.A., Johnston, D., Wilson, M.A., et al. 2002. Requirement for hippocampal CA3 NMDA receptors in associative memory recall. Science 297: 211-218.

Nakazawa, K., Sun, L.D., Quirk, M.C., Rondi-Reig, L., Wilson, M.A., and Tonegawa, S. 2003. Hippocampal CA3 NMDA receptors are crucial for memory acquisition of one-time experience. Neuron 38: 305-315.

O'Keefe, J. and Dostrovsky, J. 1971. The hippocampus as a spatial map: Preliminary evidence from unit activity in the freely-moving rat. Brain Res. 34: 171-175.

O'Keefe, J. and Nadel, L. 1978. The hippocampus as a cognitive map. Clarendon Press, Oxford, UK.

O'Keefe, J. and Speakman, A. 1987. Single unit activity in the rat hippocampus during a spatial memory task. Exp. Brain Res. 68: 1-27.

Oler, J.A. and Markus, E.J. 2000. Age-related deficits in the ability to encode contextual change: a place cell analysis. Neuroscience 10: $338-350$.

Rolls, E.T. and Kesner, R.P. 2006. A computational theory of hippocampal function, and empirical tests of the theory. Prog. Neurobiol. 79: 1-48.

Rolls, E.T. and Treves, A. 1998. Neural networks and brain function. Oxford University Press, Oxford, UK.

Shapiro, M.L., Tanila, H., and Eichenbaum, H. 1997. Cues that hippocampal place cells encode: Dynamic and hierarchical representation of local and distal stimuli. Hippocampus 7: 624-642.

Shen, J., Barnes, C.A., McNaughton, B.L., Skaggs, W.E., and Weaver, K.L. 1997. The effect of aging on experience-dependent plasticity of hippocampal place cells. J. Neurosci. 17: 6769-6782.

Skaggs, W.E. and McNaughton, B.L. 1996. Replay of neuronal firing sequences in rat hippocampus during sleep following spatial experience. Science 271: 1870-1873.

Skaggs, W.E., McNaughton, B.L., Gothard, K.M., and Markus, E.J. 1993. An information-theoretic approach to deciphering the hippocampal code. In Advances in neural information processing systems. (eds. S.J. Hanson et al.), pp. 1030-1037. Morgan Kaufman, San Mateo, CA.

Skaggs, W.E., McNaughton, B.L., Wilson, M.A., and Barnes, C.A. 1996. Theta phase precession in hippocampal neuronal populations and the compression of temporal sequences. Hippocampus 6: 149-172.

Stern, C.E., Corkin, S., Gonzalez, R.G., Guimaraes, A.R., Baker, J.R., Jennings, P.J., Carr, C.A., Sugiura, R.M., Vedantham, V., and Rosen, B.R. 1996. The hippocampal formation participates in novel picture encoding: Evidence from functional magnetic resonance imaging. Proc. Natl. Acad. Sci. 93: 8660-8665.

Tanila, H., Shapiro, M.L., and Eichenbaum, H. 1997. Discordance of spatial representation in ensembles of hippocampal place cells. Hippocampus 7: 613-623.

Treves, A. 2004. Computational constraints between retrieving the past and predicting the future, and the CA3-CA1 differentiation. Hippocampus 14: 539-556.

Treves, A. and Rolls, E.T. 1994. Computational analysis of the role of the hippocampus in memory. Hippocampus 4: 374-391.

Tse, D., Langston, R.F., Kakeyama, M., Bethus, I., Spooner, P.A., Wood, E.R., Witter, M.P., and Morris, R.G.M. 2007. Schemas and memory consolidation. Science 316: 76-82.

Vargha-Khadem, F., Gadian, D.G., Watkins, K.E., Connelly, A., Van Paesschen, W., and Mishkin, M. 1997. Differential effects of early hippocampal pathology on episodic and semantic memory. Science 277: $376-380$

Vinogradova, O.S. 2001. Hippocampus as comparator: Role of the two input and two output systems of the hippocampus in selection and registration of information. Hippocampus 11: 578-598.

Wilson, M.A. and McNaughton, B.L. 1993. Dynamics of the hippocampal ensemble code for space. Science 261: 1055-1058.

Wilson, M.A. and McNaughton, B.L. 1994. Reactivation of hippocampal ensemble memories during sleep. Science 265: 676-679.

Wood, E.R., Dudchenko, P.A., and Eichenbaum, H. 1999. The global record of memory in hippocampal neuronal activity. Nature 397: 613-616.

Yu, X., Knierim, J.J., Lee, I., and Shouval, H.Z. 2006. Simulating place field dynamics using spike timing-dependent plasticity. Neurocomputing 69: 1253-1259.

Received July 29, 2007; accepted in revised form September 5, 2007 


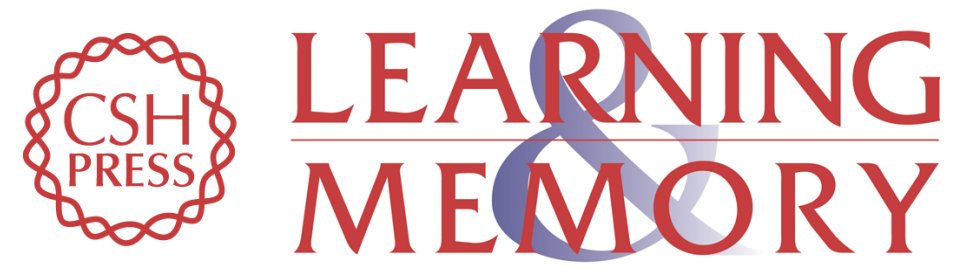

\section{The relationship between the field-shifting phenomenon and representational coherence of place cells in CA1 and CA3 in a cue-altered environment}

Inah Lee and James J. Knierim

Learn. Mem. 2007, 14:

Access the most recent version at doi:10.1101//m.706207

References This article cites 52 articles, 15 of which can be accessed free at: http://learnmem.cshlp.org/content/14/11/807.full.html\#ref-list-1

License

Email Alerting

Receive free email alerts when new articles cite this article - sign up in the box at the Service top right corner of the article or click here. 